\title{
Protective Measurements: extracting the expectation value by measuring a single particle.
}

\author{
Fabrizio Piacentini ${ }^{\mathrm{a}}$, Alessio Avella ${ }^{\mathrm{a}}$, Enrico Rebufello ${ }^{\mathrm{a}, \mathrm{b}}$, Salvatore Virzì ${ }^{\mathrm{a}, \mathrm{c}}$, Rudi Lussana ${ }^{\mathrm{d}}$, \\ Federica Villa ${ }^{\mathrm{d}}$, Alberto Tosi ${ }^{\mathrm{d}}$, Marco Gramegna ${ }^{\mathrm{a}}$, Giorgio Brida ${ }^{\mathrm{a}}$, Eliahu Cohen ${ }^{\mathrm{e}}$, Lev \\ Vaidman $^{\mathrm{f}}$, Ivo Pietro Degiovanni ${ }^{\mathrm{a}}$, and Marco Genovese ${ }^{\mathrm{a}}$ \\ aINRIM, Strada delle Cacce 91, I-10135, Torino, Italy

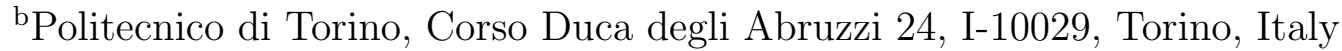 \\ cDipartimento di Fisica, Università di Torino, via Giuria 1, I-10125, Torino, Italy \\ ${ }^{\text {d} P o l i t e c n i c o ~ d i ~ M i l a n o, ~ D i p a r t i m e n t o ~ d i ~ E l e t t r o n i c a, ~ I n f o r m a z i o n e ~ e ~ B i o i n g e g n e r i a, ~ P i a z z a ~}$ \\ Leonardo da Vinci 32, 20133, Milano, Italy \\ ${ }^{\text {e} H . H . ~ W i l l s ~ P h y s i c s ~ L a b o r a t o r y, ~ U n i v e r s i t y ~ o f ~ B r i s t o l, ~ T y n d a l l ~ A v e n u e, ~ B r i s t o l, ~ B S 8 ~ 1 T L, ~ U . K . ~}$ \\ ${ }^{\mathrm{f}}$ Raymond and Beverly Sackler School of Physics and Astronomy, Tel-Aviv University, \\ Tel-Aviv 6997801, Israel
}

\begin{abstract}
In quantum mechanics, the eigenvalues and their corresponding probabilities specify the expectation value of a physical observable, which is known to be a statistical property related to large ensembles of particles. In contrast to this paradigm, we demonstrate a unique method allowing to extract the expectation value of a single particle, namely, the polarisation of a single protected photon, with a single experiment. This is the first realisation of quantum protective measurements.
\end{abstract}

Keywords: foundations of quantum mechanics, quantum optics, quantum metrology, single photons, expectation value, protective measurements

\section{INTRODUCTION}

Despite its success in predicting experimental results, there is no consensus about the foundations of quantum mechanics. ${ }^{1-8}$

In this framework, measurement plays an undisputed and fundamental role, ${ }^{9}$ since it's the process in which some of the distinctive traits of the quantum world with respect to the classical one appear, e.g. the collapse of quantum states due to a strong (projective) measurement. This collapse causes the impossibility to measure non-commuting observables onto the same quantum state. In the last decades, new quantum measurement paradigms emerged, e.g. weak measurement, introduced in ${ }^{10,11}$ and firstly realized in, ${ }^{12-14}$ in which the coupling strength between quantum state and measurement system is weak enough to prevent the quantum state collapse at the cost of extracting only a small amount of information from a single measurement. Weak measurements outcomes can be "anomalous" (imaginary and/or unbounded) values, being the imaginary part related to the disturbance of the measuring pointer during the measurement. ${ }^{15}$ This behavior inspired significant analysis on quantum measurement and its meaning, ${ }^{16-19}$ foundational problems,${ }^{20}$ macrorealism ${ }^{21}$ and contextuality. ${ }^{22,23}$

In quantum metrology and quantum technologies development, they allow high-precision measurements (at least in presence of specific noises ${ }^{24}$ ), as the tiny spin Hall effect ${ }^{14}$ or small beam deflections, ${ }^{25}$ and quantum states characterisation. ${ }^{26}$ In addition, the absence of wave function collapse in weak measurements allows performing sequential measurements of non-commuting observables onto the same quantum state. ${ }^{27}$

A second example of innovative measurement paradigm is represented by Protective Measurement. Originally proposed within the debate on the reality of the wave function, ${ }^{28}$ Protective Measurement was deeply 
studied in many theoretical works ${ }^{29-34}$ and recently realized for the first time. ${ }^{35}$ It links the weak interaction typical of weak measurements with some protection mechanism of the measured state. Although a hot debate topic from the quantum foundations perspective, ${ }^{36-43}$ protective measurements have demonstrated exceptional measurement capability, giving the possibility of extracting the quantum expectation value of an observable by a single measurement on a single (protected) particle, ${ }^{35}$ a task forbidden in the traditional quantum measurement framework. Indeed, complete description of a quantum system allows predicting just the (eigen)values spectrum and related probabilities for the measurement as outcomes of a measured observable. To each observable $A$ and quantum state $|\psi\rangle$ we can associate a definite number, called expectation value: $\langle A\rangle=\langle\psi|A| \psi\rangle=\sum_{i} p_{i} a_{i}$, where $p_{i}$ is the probability to obtain the outcome $a_{i}$ as a result of the measurement of $A$. The expectation value is a statistical property of the system, meaning that it can be extracted only by repeated measurements over an ensamble of identical systems. Here, we show that, in some special cases, the expectation value can be extracted by performing only a single measurement, thanks to the measurement procedure called protective measurement. ${ }^{28,44}$

\section{THEORY}

Let us suppose that we want to measure the expectation value of an observable $A$. To do so, we use the von Neumann protocol, in which measuring an observable $A$ in a state $|\psi\rangle$ requires an interaction Hamiltonian

$$
H_{\text {int }}=g A \otimes P
$$

that couples the measured system to the measuring device by means of the coupling constant $g$, where $P$ is the momentum conjugate to the pointer variable $Q$ releated to the device. The initial distribution of our system with respect to the pointer $Q$ is $\phi_{0}(q)=e^{-q / 2 \sigma}$. In the traditional projective measurement framework, the final pointer state is in a superposition of $\phi_{i}(q)=\phi_{0}\left(q-g a_{i}\right)$ wavefuncions, with $a_{i}$ being the eigenvalues of $A$. For strong interactions $(g / \sigma>>1)$, the eigenstates of $A$ are distinguishible, thus it is possible to statistically obtain the expectation value of $A\left(\langle A\rangle=\sum_{i} p_{i} a_{i}\right.$, being $p_{i}$ the ratio of $a_{i}$ outcomes obtained).

Things change when protective measurements ${ }^{45}$ are involved. They combine a weak von Neumann coupling ${ }^{46}$ $(g / \sigma<<1)$ to a "protection" mechanism preserving state from (small) decoherence due to the von Neumann interaction. Such protection can be implemented by means of an adiabatic potential (passive protection), when the state is in a nondegenerate energy eigenstate, ${ }^{47}$ or via quantum Zeno effect (active protection). ${ }^{48}$ In the second case, the protection consists of a projection onto the initial state $|\psi\rangle$ in cascade to the weak interaction, i.e.:

$$
\Pi_{\psi}=|\psi\rangle\langle\psi|
$$

this leads us to:

$$
|\psi\rangle\left\langle\psi\left|\exp \left(-i H_{\text {int }}\right)\right| \psi\right\rangle|\phi(q)\rangle \approx|\psi\rangle|\phi(x-g\langle A\rangle)\rangle
$$

for $g / \sigma<<1$.

Hence, the expectation value of the measured observable can be extracted directly from a measurment of the pointer. Furthermore, the measured state $|\psi\rangle$ is not changed by the protective measurement procedure. ${ }^{49}$

In the following, we experimentally demonstrate how protective measurements allow to obtain $\langle A\rangle$ not as a statistical average, but as a reading of a measurement device coupled to a single system.

\subsection{Our implementation}

In our experiment, we produce single photons prepared in the quantum states $|\psi(\theta)\rangle=\cos (\theta)|H\rangle+\sin (\theta)|V\rangle$, where $H(V)$ is the horizontal(vertical) polarisation. In our scheme, we want to measure the expectation value of the polarisation $A=|H\rangle\langle H|-| V\rangle\langle V|$ of these single photons.

Our implementation of protective measurement consists in a series of weak von Neumann couplings, in which the polarisation $A$ is weakly coupled to the transverse momentum $P$ of the photon, each followed by the protection $\Pi_{\psi}$. 
Without protections, we can imagine this procedure as an optical Stern-Gerlach experiment ${ }^{50}$ in which $K$ weak interactions result into a strong interaction (with strenght $g^{\prime}=K g$ ), completely separating the two polarisation components. In this case, the expectation value of the polarisation is calculated with the statistical formula:

$$
\langle A\rangle=\frac{N_{H}-N_{V}}{N_{H}+N_{V}}
$$

where $N_{H(V)}$ is the number of counts observed in the region corresponding to the horizontal(vertical) polarisation.

On the other hand, when the active protection carried out by the projector $\Pi_{\psi}$ is implemented, the combined effect of the $K$ steps of weak interaction and protection causes a shift of the pointer proportional to the expectation value of the polarisation $\langle A\rangle$. For this reason, the expectation value can be extracted from the position of each photon impinging on the measurement device:

$$
\langle A\rangle=\frac{x-x_{0}}{a}
$$

with $a=g^{\prime} / 2$ and $x_{0}=\frac{x_{H}+x_{V}}{2}$, being $x_{H}\left(x_{V}\right)$ the center of the horizontally-(vertically-)polarised photon distribution in the non-proteted case.

While, in the non-protected case, the expectation value can be extracted statistically only by measuring an ensamble of identical photons, in the protected case each photon carries information about the expectation value of its polarisation, allowing to extract the polarisation expectation value even by a single experiment on a single photon.

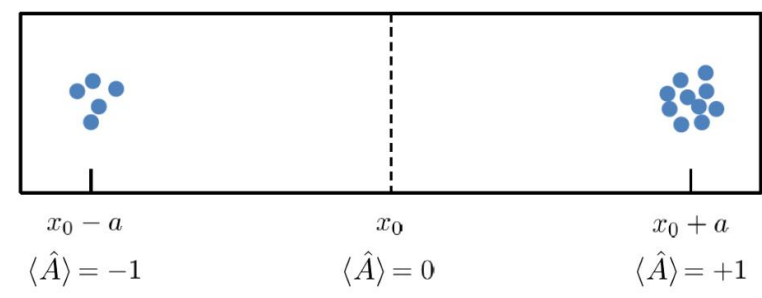

(a) Stern-Gerlach like measurements: the photons fall in one of the two regions corresponding to the $H$ and $V$ polarisations.

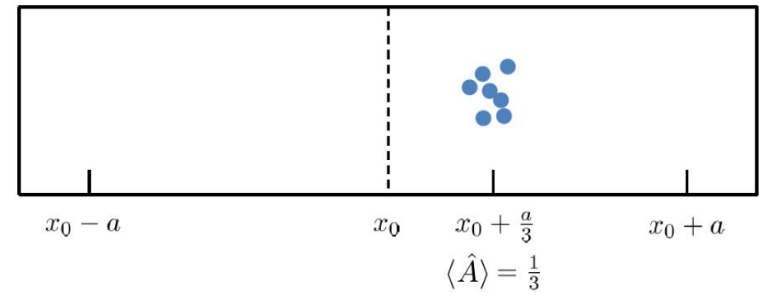

(b) Protective measurements: the photons fall in region whose center is located proportionally to the expectation value of the polarization.

Figure 1: Projective vs protective measurement

\section{EXPERIMENT}

\subsection{Setup}

The experimental setup (Fig.2)can be divided in two part: the single photon generation stage and the preparation and measurement stage. In the first one, a Ti:Sapphire mode-locked laser at $796 \mathrm{~nm}$, frequency-doubled by second armonic generation (SHG), enters a $\mathrm{LiIO}_{3}$ non-linear crystal, where type-I parametric down-conversion occurs. ${ }^{51}$ This way, we obtain a heralded single photon source ${ }^{52}$ in which an idler photon $\left(\lambda_{i}=920 \mathrm{~nm}\right)$, properly filtered and coupled to a single mode fiber (SMF), heralds the presence of its correlated signal photon $\left(\lambda_{s}=702 \mathrm{~nm}\right)$. Such heralded photon is filtered, SMF-coupled and addressed to the preparation and measurement stage. Here, the single photon is collimated in a gaussian spatial distribution and then prepared in the polarisation $|\psi(\theta)\rangle=\cos (\theta)|H\rangle+\sin (\theta)|V\rangle$ by a polarising beam splitter followed by a half-wave plate. The protective measurement protocol is realised by $K$ interaction-protection steps. In our implementation, the weak interaction is performed by a pair of thin birefringent crystals: the first one creates a small shift between the two orthogonal polarisation components, while the second one compensates the phase and the temporal walk-off introduced by the first crystal. The protection, instead, is carried by a thin-film polariser projecting the single photon onto the initial state $|\psi(\theta)\rangle$. Ideally, protective measurements are composed of an infinite number of steps, i.e. $K \rightarrow \infty$. 


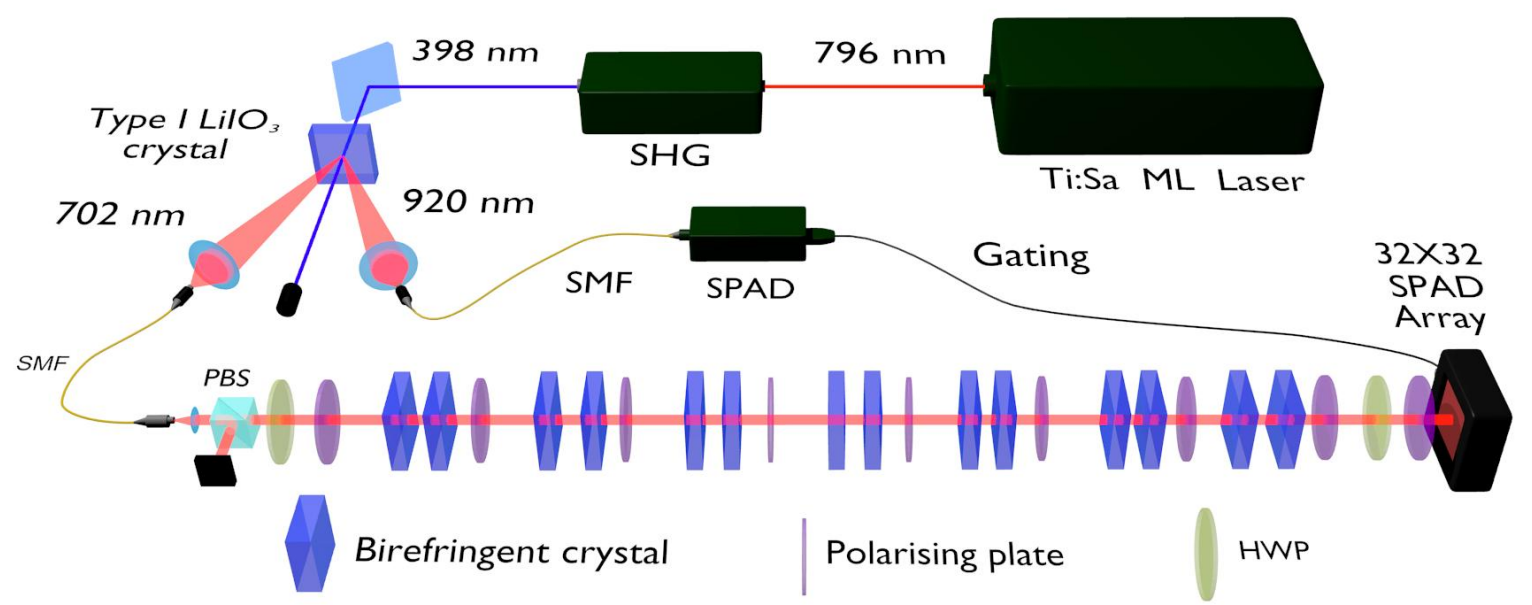

Figure 2: Experimental setup

In our experiment we implement $K=7$ interaction-protection steps, the maximun number allowed by our setup limitations. At the end of these steps, the photons are detected by a spatial-resolving single-photon detector, i.e. a prototype array of $32 \times 32 \mathrm{SPADs}^{53}$ (Single-Photons Avalanche Diode).

By removing the thin-film polarisers, the effect of the birefringent crystal pairs series induce the spatial separation between orthogonal polarisations. Thus, we can perform a traditional (projective) measurement by counting the photons detected in the spatial-resolving detector sectors corresponding to the two polarisations. This way, we are able to compare expectation values of $A$ obtained with protective and projective measurements.

\subsection{Results}

In Fig.3 we compare the results of the two measurements protocols, each performed over the same initial polarisation state $|\psi(17 \pi / 60)\rangle=0.629|H\rangle+0.777|V\rangle$ in a time interval of $1200 \mathrm{~s}$.

Fig.s 3.a,b show the histograms of heralded photons detected with traditional measurement and protective measurement, respectively. In Fig.s 3.c,d we can see the same distributions contour plots, in order to show the different spatial sectors of photon-detection between projective and protective measurements.

As in a standard Stern-Gerlach experiment, in the projective case (Fig.s 3.a,c) we have two regions of photondetection corresponding to the possible eigenvalues of $A$. The experimental expectation value $\langle A\rangle$ (calculated with (4)) from these distributions (dark counts subtracted) is $\left\langle A_{17 \pi / 60}\right\rangle=-0.21(4)$, in agreement with the theoretical value $\left\langle A_{17 \pi / 60}^{t h}\right\rangle=-0.208$. In the protective case, we find a single photon counts distribution centered in the position corresponding to the expectation value $\left\langle A_{17 \pi / 60}\right\rangle=-0.19(2)$, in ageement both with the teoretical value and the projective one.

This result shows that, with protective measurements, it is possible to extract the expectation value of the measured observable even by performing a single experiment on a single (protected) quantum system. This property clearly emerges when looking at Fig.s 4.a,b, reporting the results obtained with the two measurement techniques in a few photons experiment $(N=17$ detections for the protective measurement, and $N=14$ for the projective one).

In Fig. 4.a is shown the projective case. The red circles represent the FWHM (Full Width at Half Maximum) of the two distributions in Fig.s 3.a,c. The first detected photon in the run is signified with a yellow pixel.

In Fig. 4.b (protective case), photons are detectected close to the position corresponding to $\langle A\rangle$ (despite the non-negligible dark count level of our non-ideal SPAD array, likely responsible for the detection events outside the circles). As in Fig. 4.a, the red circle is the FWHM of the distribution in Fig.s 3.b,d and the white pixel represents the first detected photon. 


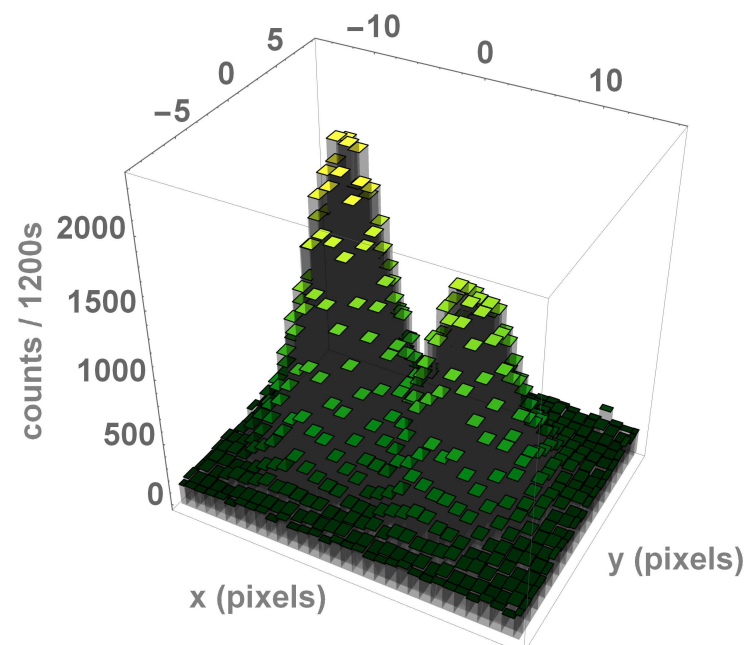

(a) Projective measurement counts histogram.

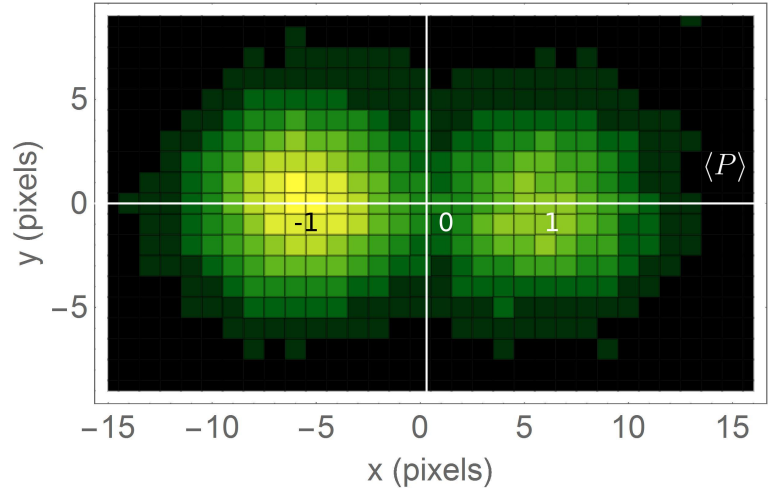

(c) Projective measurement contour plot.

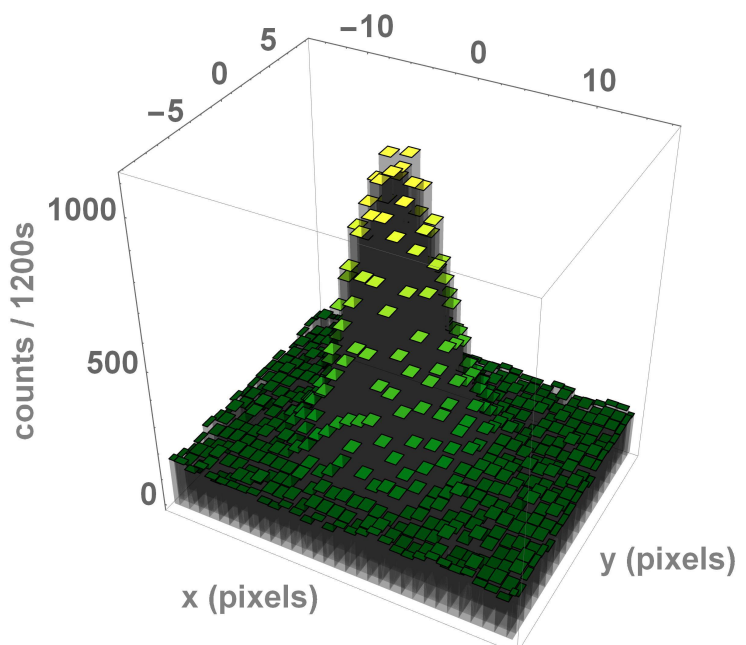

(b) Protective measurement counts histogram.

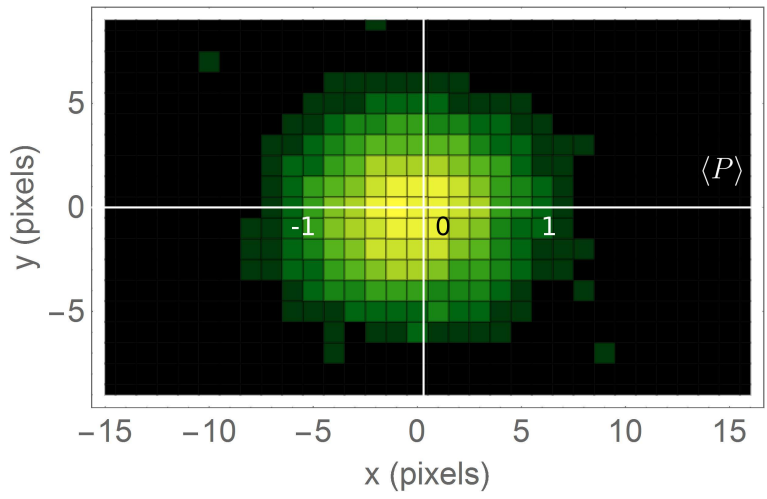

(d) Projective measurement contour plot.

Figure 3: Results of the two measurement protocols onto the initial polarisation state $|\psi(17 \pi / 60)\rangle$.

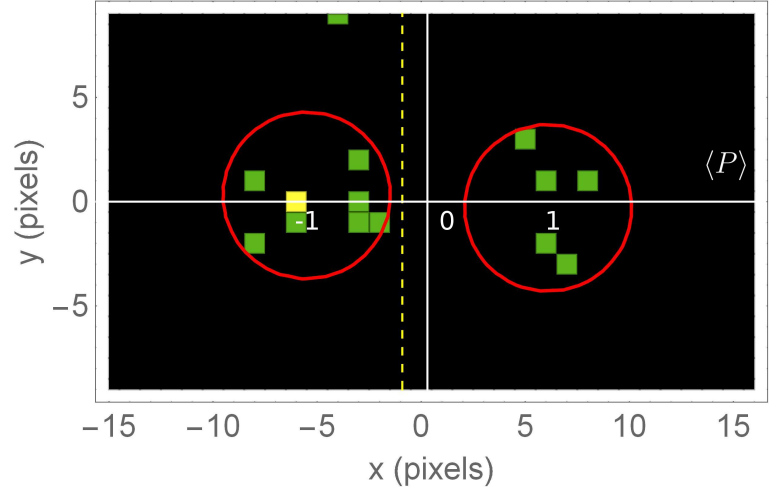

(a) Projective case.

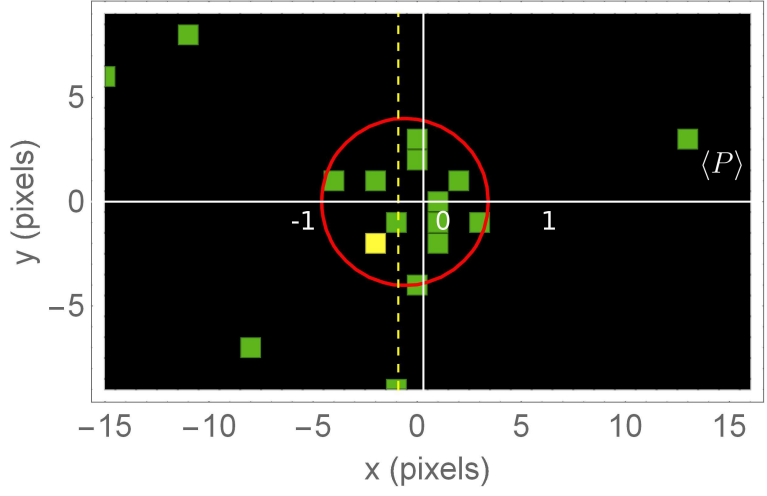

(b) Protective case

Figure 4: Results of the two measurement protocols with few photons. 
We see that, although the white pixel in Fig. 4.b provides a good estimate of $\langle A\rangle$, that cannot be said for the white pixel of Fig. 4.a: this demonstrates the protective measurements capability of extracting the expectation value $\langle A\rangle$ from just few detection events (even one, in the ideal case).

\section{CONCLUSIONS}

This is the first experimental realisation of protective measurements. ${ }^{35}$ The experiment shows that a single photon detection can provide reliable information regarding the expectation value of the measured observable (polarisation, in our case), supposed to be only a statistical property to be extracted from an ensemble of identical quantum systems.

\section{ACKNOWLEDGMENTS}

This work has received funding from the European Union's Horizon 2020 and the EMPIR Participating States in the context of the project EMPIR-14IND05 "MIQC2", and from the INRIM "Seed" project "GeQuM". E.C. was supported by ERC AdG NLST. L.V. acknowledges support of the Israel Science Foundation Grant No. 1311/14 and the German-Israeli Foundation for Scientific Research and Development Grant No. I-1275-303.14.

\section{REFERENCES}

[1] Popescu, S., "Nonlocality beyond quantum mechanics," Nature Physics 10, 264-270 (2014).

[2] Genovese, M., "Research on hidden variable theories: A review of recent progresses," Physics Reports 413, 319-396 (2005).

[3] G Brida, M Genovese, F. P., "Experimental local realism tests without fair sampling assumption," The European Physical Journal D 44, 577-580 (2007).

[4] Polyakov, S. V., Piacentini, F., Traina, P., Degiovanni, I. P., Migdall, A., Brida, G., and Genovese, M., "Practical implementation of a test of event-based corpuscular model as an alternative to quantum mechanics," Foundations of Physics 43, 913-922 (Aug 2013).

[5] A Avella, M. G., "50 years of experimental bell inequalities: A short introduction," Journal of Advanced Physics 4, 233-235 (2015).

[6] G. Brida, M. G. and Piacentini, F., "Experimental local realism tests without fair sampling assumption," F. Eur. Phys. J. D 44, 577 (2007).

[7] Vaidman, L., "Many-worlds interpretation of quantum mechanics," in [The Stanford Encyclopedia of Philosophy], Zalta, E. N., ed., Metaphysics Research Lab, Stanford University, fall 2016 ed. (2016).

[8] Vaidman, L., "Past of a quantum particle," Phys. Rev. A 87, 052104 (May 2013).

[9] Genovese, M., "Interpretations of quantum mechanics and the measurement problem," Adv. Sci. Lett 3, 249-258.

[10] Aharonov, Y., Albert, D. Z., and Vaidman, L., "How the result of a measurement of a component of the spin of a spin-1/2 particle can turn out to be 100," Phys. Rev. Lett. 60, 1351-1354 (Apr 1988).

[11] Tamir, B. and Cohen, E., "Introduction to weak measurements and weak values," Quanta 2(1) (2013).

[12] Ritchie, N. W. M., Story, J. G., and Hulet, R. G., "Realization of a measurement of a "weak value"," Phys. Rev. Lett. 66, 1107-1110 (Mar 1991).

[13] Pryde, G. J., O'Brien, J. L., White, A. G., Ralph, T. C., and Wiseman, H. M., "Measurement of quantum weak values of photon polarization," Phys. Rev. Lett. 94, 220405 (Jun 2005).

[14] Hosten, O. and Kwiat, P., "Observation of the spin hall effect of light via weak measurements," Science 319(5864), 787-790 (2008).

[15] Dressel, J. and Jordan, A. N., "Significance of the imaginary part of the weak value," Phys. Rev. A 85, 012107 (Jan 2012).

[16] Vaidman, L., "Weak value controversy," arXiv preprint arXiv: arXiv:1703.08870 (2017).

[17] Lev Vaidman, Alon Ben-Israel, J. D. L. K. M. W. J. M. C. S. R. B. H. W., "Weak value beyond conditional expectation value of the pointer readings," arXiv preprint: arXiv:1611.03989 (2016). 
[18] Aharonov, Y., Cohen, E., and Elitzur, A. C., "Foundations and applications of weak quantum measurements," Phys. Rev. A 89, 052105 (May 2014).

[19] Aharonov, Y., Cohen, E., and Elitzur, A. C., "Can a future choice affect a past measurements outcome?," Annals of Physics 355, 258 - 268 (2015).

[20] Aharonov, Y., Botero, A., Popescu, S., Reznik, B., and Tollaksen, J., "Revisiting hardy's paradox: counterfactual statements, real measurements, entanglement and weak values," Physics Letters A 301(3), 130 138 (2002).

[21] Avella, A., Piacentini, F., Borsarelli, M., Barbieri, M., Gramegna, M., Lussana, R., Villa, F., Tosi, A., Degiovanni, I. P., and Genovese, M., "Anomalous weak values and the violation of a multiple-measurement leggett-garg inequality," Phys. Rev. A 96, 052123 (Nov 2017).

[22] Pusey, M. F., "Anomalous weak values are proofs of contextuality," Phys. Rev. Lett. 113, 200401 (Nov 2014).

[23] Piacentini, F., Avella, A., Levi, M. P., Lussana, R., Villa, F., Tosi, A., Zappa, F., Gramegna, M., Brida, G., Degiovanni, I. P., and Genovese, M., "Experiment investigating the connection between weak values and contextuality," Phys. Rev. Lett. 116, 180401 (May 2016).

[24] Dressel, J., Malik, M., Miatto, F. M., Jordan, A. N., and Boyd, R. W., "Colloquium: Understanding quantum weak values: Basics and applications," Rev. Mod. Phys. 86, 307-316 (Mar 2014).

[25] Resch, K. J., "Amplifying a tiny optical effect," Science 319(5864), 733-734 (2008).

[26] Jeff Z. Salvail, Megan Agnew, A. S. J. E. B. J. L. . R. W. B., "Full characterization of polarization states of light via direct measurement," Nature Photonics 7, 316-321 (2013).

[27] Piacentini, F., Avella, A., Levi, M. P., Gramegna, M., Brida, G., Degiovanni, I. P., Cohen, E., Lussana, R., Villa, F., Tosi, A., Zappa, F., and Genovese, M., "Measuring incompatible observables by exploiting sequential weak values," Phys. Rev. Lett. 117, 170402 (Oct 2016).

[28] Aharonov, Y. and Vaidman, L., "Measurement of the schrodinger wave of a single particle," Physics Letters A 178(1), 38 - 42 (1993).

[29] Aharonov, Y., Englert, B.-G., and Scully, M. O., "Protective measurements and bohm trajectories," Physics Letters A 263(3), 137 - 146 (1999).

[30] Gao, S., [Protective Measurement and Quantum Reality: Towards a New Understanding of Quantum Mechanics], Cambridge University Press (2015).

[31] Schlosshauer, M., "Measuring the quantum state of a single system with minimum state disturbance," Phys. Rev. A 93, 012115 (Jan 2016).

[32] Aharonov, Y. and Vaidman, L., "Protective measurements of two-state vectors," In: Cohen R.S., Horne M., Stachel J. (eds) Potentiality, Entanglement and Passion-at-a-Distance. Boston Studies in the Philosophy of Science 194 (1997).

[33] Uffink, J., "How to protect the interpretation of the wave function against protective measurements," Phys. Rev. A 60, 3474-3481 (Nov 1999).

[34] Hari Dass, N. D. and Qureshi, T., "Critique of protective measurements," Phys. Rev. A 59, 2590-2601 (Apr 1999).

[35] F. Piacentini, e. a., "Determining the quantum expectation value by measuring a single photon," Nature Physics 13, 11911194 (2017).

[36] M. Ringbauer, B. Duffus, C. B. E. G. C. A. G. W. and Fedrizzi, A., "Measurements on the reality of the wavefunction," Nature Physics 11, 249-254 (2015).

[37] Colbeck, R. and Renner, R., "Is a systems wave function in one-to-one correspondence with its elements of reality?," Phys. Rev. Lett. 108, 150402 (2012).

[38] Y. Aharonov, J. Anandan, L. V., "Meaning of the wave function," Phys. Rev. A 47, 4616 (1993).

[39] Rovelli, C., "Comment on meaning of the wave function," Phys. Rev. A 50, 2788 (1994).

[40] D'Ariano, G. M. and Yuen, H. P., "Impossibility of measuring the wave function of a single quantum system," Phys. Rev. Lett. 76, 2832-2835 (Apr 1996).

[41] Hardy, L., "Are quantum states real?," Int. J. Mod. Phys. B 27, 1345012 (2013).

[42] Pusey, M. F., B. J. . R. T., "On the reality of the quantum state," Nat. Phys. 8, 475-478 (2012).

[43] Unruh, W. G., "Reality and measurement of the wave function," Phys. Rev. A 50, 882-887 (Jul 1994). 
[44] Y. Aharonov, J. Anandan, L. V., "The meaning of protective measurements," L. Found Phys 26, 117 (1996).

[45] Vaidman, L., "Protective measurements," In: Compendium of Quantum Physics (2009).

[46] Fabrizio Piacentini, Alessio Avella, M. G. R. L. F. V. A. T. G. B. I. P. D. M. G., "Investigating the effects of the interaction intensity in a weak measurement," arXiv preprint arXiv:1709.04869 (2017).

[47] Aharonov, Y. and Vaidman, L., "The schrodinger wave is observable after all!," In Quantum Control and Measurement (1993).

[48] Misra, B. and Sudarshan, E. C. G., "The zeno's paradox in quantum theory," Journal of Mathematical Physics 18(4), 756-763 (1977).

[49] Y. Aharonov, J. Anandan, L. V., "Protective measurements," Found Phys 26(117) (1996).

[50] T. Sleator, T. Pfau, V. B. O. C. J. M., "Experimental demonstration of the optical stern-gerlach effect," Phys. Rev. Lett. 68, 1996-1999 (Mar 1992).

[51] Avella, A., "Theory of parametric down conversion in bulk non-linear crystals: An introduction," Journal of Advanced Physics 4(3), 252-262 (2015).

[52] G. Brida, I. P. Degiovanni, M. G. F. P. P. T. A. D. F. A. T. A. B. S. C. S. A. G. M. G. S. V. P. A. M. and Giudice, A., "An extremely low-noise heralded single-photon source: A breakthrough for quantum technologies," Appl. Phys. Lett. 101 (2012).

[53] F. Villa, R. Lussana, D. B. S. T. A. T. F. Z. A. D. M. D. C. D. D. S. W. W. B., "Cmos imager with 1024 spads and tdes for single-photon timing and 3-d time-of-flight," IEEE Journal of Selected Topics in Quantum Electronics 20, 364-373 (Nov 2014). 